\title{
The brief negative symptom scale: validation of the German translation and convergent validity with self-rated anhedonia and observer-rated apathy
}

Martin Bischof ${ }^{1 *}$, Caitriona Obermann ${ }^{1}$, Matthias N. Hartmann ${ }^{1,2}$, Oliver M. Hager ${ }^{1,2}$, Matthias Kirschner ${ }^{1}$, Agne Kluge ${ }^{1}$, Gregory P. Strauss ${ }^{3}$ and Stefan Kaiser ${ }^{2}$

\begin{abstract}
Background: Negative symptoms are considered core symptoms of schizophrenia. The Brief Negative Symptom Scale (BNSS) was developed to measure this symptomatic dimension according to a current consensus definition. The present study examined the psychometric properties of the German version of the BNSS. To expand former findings on convergent validity, we employed the Temporal Experience Pleasure Scale (TEPS), a hedonic self-report that distinguishes between consummatory and anticipatory pleasure. Additionally, we addressed convergent validity with observer-rated assessment of apathy with the Apathy Evaluation Scale (AES), which was completed by the patient's primary nurse.

Methods: Data were collected from 75 in- and outpatients from the Psychiatric Hospital, University Zurich diagnosed with either schizophrenia or schizoaffective disorder. We assessed convergent and discriminant validity, internal consistency and inter-rater reliability.

Results: We largely replicated the findings of the original version showing good psychometric properties of the BNSS. In addition, the primary nurses evaluation correlated moderately with interview-based clinician rating. BNSS anhedonia items showed good convergent validity with the TEPS.

Conclusions: Overall, the German BNSS shows good psychometric properties comparable to the original English version. Convergent validity extends beyond interview-based assessments of negative symptoms to self-rated anhedonia and observer-rated apathy.
\end{abstract}

Keywords: Schizophrenia, Negative symptoms, Rating scale, Assessment

\section{Background}

The negative symptoms of schizophrenia consist of flattened affect, alogia, social withdrawal, avolition, and anhedonia [1]. They account to a large extent for impairments of work and social functioning, as well as longterm morbidity [2-4]. Today, pharmacological and psychosocial interventions have limited efficacy for reducing negative symptoms $[5,6]$.

\footnotetext{
*Correspondence: martinbischof@gmail.com; martin.bischof@puk.zh.ch 'Department of Psychiatry, Psychotherapy and Psychosomatics, Psychiatric Hospital, University of Zurich, Lenggstrasse 31, 8032 Zurich, Switzerland
} Full list of author information is available at the end of the article
The reliable and valid assessment of negative symptoms is critical for the development of pathophysiological models and effective treatments [7]. Since the introduction of quantitative assessment of negative symptoms in the early 1980s, concepts of negative symptoms have undergone subtle but important changes [8,9]. Most importantly, this includes the distinction of two main factors of negative symptoms apathy/avolition and diminished expression $[1,10]$. Other important shifts include the definition of cognitive dysfunction as a separate symptom dimension, the distinction between objective and subjective aspects of apathy/avolition, and the separation of consummatory and anticipatory anhedonia [9]. 
A consensus conference in 2005 under the auspices of the National Institute of Mental Health (NIMH) has provided an integration of these developments into a common conception of negative symptoms [9]. A main outcome of this consensus conference was the agreement that new instruments would have to be developed in order to fully cover this modern conceptualization of negative symptoms. Subsequently, two scales were developed - the Clinical Assessment Interview for Negative Symptoms (CAINS) [11] and the Brief Negative Symptom Scale (BNSS) [12]. The BNSS was explicitly intended for easy application in the context of clinical trials or experimental psychopathology studies. The BNSS is a 13-item instrument, which allows rapid assessment of negative symptoms based on a semi-structured interview. The authors were able to establish excellent psychometric properties in a series of validation studies [12-14]. Successful translations have been accomplished for Spanish and Italian versions of the BNSS, which also demonstrate excellent psychometric properties $[15,16]$.

The present study aimed to establish reliability and validity of the German version of the BNSS. In addition, we expanded previous investigations of convergent validity by discriminating between self-reported anticipatory and consummatory pleasure and by including observerbased measures of apathy/avolition. First, in order to assess whether the BNSS anhedonia subscales capture the self-rated subjective experience of anhedonia, we included the Temporal Experience of Pleasure Scale. Second, we assessed the relationship between BNSS ratings and observer-rated apathy/avolition by including the Apathy Evaluation Scale rated by the primary nurse. We also included the Modified Simpson-Angus Scale (MSAS) as a measure for extrapyramidal side effects in the estimation of divergent validity.

\section{Methods \\ Participants}

Seventy-five individuals meeting Diagnostic and Statistical Manual of Mental Disorders, Fourth Edition (DSM-IV) criteria for schizophrenia $(n=65)$ or schizoaffective disorder ( $n=10$, not currently in a mood episode) were included in the study. The local Ethics committee approved the study, and all participants gave written informed consent.

Inclusion criteria were: (1) outpatient or inpatient treatment at the Psychiatric Hospital, University of Zurich, (2) a clinical diagnosis schizophrenia or schizoaffective disorder and (3) age from 18 to 65 years. Importantly, all patients had to be clinically stable, i.e. they did not show positive symptoms of more than moderate severity (see exclusion criteria below) and received a constant dose of antipsychotic medication for at least two weeks prior to testing. Exclusion criteria were: (1) daily lorazepam dosage $>1 \mathrm{mg}$, (2) positive symptoms of moderate severity (Positive and Negative Syndrome Scale (PANSS) [17]; any positive subscale item score $>4$ ), (3) history of head injury or neurological disorder, (4) insufficient German fluency (5) additional DSM-IV axis1 or axis-2 diagnostic criteria (according to the treating clinician). To confirm axis-1 diagnosis in patients and exclude comorbid axis-1 disorders, we used the MiniInternational Neuropsychiatric Interview [18]. All patients were treated with second-generation antipsychotic medication.

Patients were either inpatients at the end of their hospitalization $(n=49)$ or outpatients $(n=26)$ treated at the Psychiatric Hospital, University of Zurich. Please note that the average inpatient stay for patients with schizophrenia in Swiss psychiatric hospitals is above 40 days [19]; as a consequence a lot of of our inpatients would be treated as outpatients in different healthcare systems. Notably, inpatients took part in a multimodal treatment program and were encouraged to participate in activities outside the hospital, which permitted assessment of apathy/avolition symptoms. The The treatment setting of our participants regularly includes a morningmeeting after breakfast, activation groups and various combinations of work-, occupational-, sports- and artstherapies. These therapies take place from Monday to Friday during mornings and afternoons. Thus, the inpatient treatment program is comparable to a day-care setting. In addition to the group-based therapies, patients have individual sessions with a physician and/or a psychologist three times a week. Demographic information is presented in Table 1.

\section{Procedures}

The primary instrument of interest was the Brief Negative Symptom Scale (BNSS) [12]. As mentioned in the background section, the scale allows a rapid and reliable assessment of negative symptoms. It consists of 13 items and 6 subscales (anhedonia, distress, avolition, blunted affect and alogia, see Table 2) and can be split into the two different factors motivation and pleasure and emotional expressivity [14]. The scale includes a comprehensive manual, a workbook and a score sheet, which all have been translated into German. Negative symptoms were also assessed with the Scale for the Assessment of Negative Symptoms (SANS) [8], which is a widely used scale including 4 subscales (affective flattening/blunting, alogia, avolition/apathy and anhedonia/asociality).

The Global Assessment of Functioning scale (GAF) [20] and the Personal and Social Performance Scale (PSPS) [21] both measure functioning. However, the latter excludes psychopathological aspects and is therefore a more specific instrument for functioning. The Calgary Depression Scale for Schizophrenia (CDSS) [22] is an interview-based instrument that has been 
Table 1 Demographics $(n=75)$

\begin{tabular}{|c|c|c|}
\hline & Mean & (SD) \\
\hline Age & 31.5 & 10.9 \\
\hline Education (years) & 11.6 & 2.9 \\
\hline Duration of illness (months) & 133.2 & 224.1 \\
\hline Number of hospitalizations & 4.7 & 3.9 \\
\hline$\%$ male & 74.7 & \\
\hline$\%$ inpatients (vs outpatients) & 65.3 & \\
\hline $\begin{array}{l}\text { \% Diagnosis Schizophrenia (vs Schizoaffective) } \\
\text { Antipsychotic treatment }\end{array}$ & 86.7 & \\
\hline$\%$ second generation & 92.0 & \\
\hline$\%$ first and second generation & 4.0 & \\
\hline$\%$ none & 4.0 & \\
\hline PANSS total & 50.8 & 12.3 \\
\hline PANSS positive factor ${ }^{a}$ & 1.8 & 0.7 \\
\hline PANSS negative factor ${ }^{a}$ & 2.3 & 1.0 \\
\hline PANSS disorganized factor ${ }^{a}$ & 1.7 & 0.7 \\
\hline PANSS excited factor ${ }^{a}$ & 1.2 & 0.4 \\
\hline PANSS depressed factor ${ }^{a}$ & 1.7 & 0.8 \\
\hline CDSS total & 1.8 & 2.2 \\
\hline GAF total & 55.9 & 11.6 \\
\hline
\end{tabular}

developed to identify depressive symptoms in patients with schizophrenia. The Positive and Negative Syndrome Scale (PANSS) [17] is a 30-item scale that is split into three dimensions (negative symptoms, positive symptoms and general psychopathology including depression and anxiety) and allows to capture a broad range of symptomatology. However, based on recent findings about the underlying structure of the PANSS, a 5-factor model (positive, negative, disorganized/concrete, excited and depressed) described by Wallwork et al. was used for all calculations [23].

The five clinical raters, who completed ratings for all scales, were trained and regularly supervised by the senior author. Raters were trained individually, which included a step-by-step instruction on how to use the scale, joint ratings of videos and live interviews and conduction of supervised interviews. To increase interrater reliability, raters were additionally trained in video-based group sessions. Three of them have a masters-degree in clinical psychology and two were psychiatrists in training.

The German version of the BNSS is based on a translation process including forward translation, back translation, and reconciliation of back translation with the BNSS developers. This process therefore met the highest industry standards. To extend the evaluation of convergent/discriminant validity beyond the original English BNSS studies, the Apathy Evaluation Scale (AES-C) [24] was administered by the primary nurse for the inpatients only. The AES-C was established to measure apathy for neurological and psychiatric patients. The Temporal Experience of Pleasure Scale (TEPS) [25] was filled out by the patients for anhedonia self-report. It allows to distinguish consummatory and anticipatory anhedonia which is specifically important in the role of schizophrenia. Finally, the Modified Simpson-Angus Scale (MSAS) [26] was completed by the physician raters to evaluate extrapyramidal symptoms.

To assess inter-rater reliability, two different assessors rated 27 patients independently within the same session. The raters attended the same interview in person but were then separated to complete ratings.

\section{Statistical analysis}

To calculate correlations for convergent and discriminant validity, Pearson's $r$ was computed. A partial correlation between negative symptoms (BNSS total) and functioning (GAF) was conducted in order to control the influence of common sources of secondary negative symptoms on this association. Reliability was calculated with Cronbach's alpha for internal consistency and Intraclass Correlation Coefficient (ICC) for inter-rater agreement. Statistical analyses were performed using IBM SPSS Statistics Version 21. A test for significant differences in the magnitude of the correlation between the BNSS and the TEPS pleasure scales was performed using Steiger's method implemented in the online tool http:// quantpsy.org/corrtest/corrtest2.htm [27, 28].

\section{Results}

\section{Descriptive statistics and distribution of scores}

Descriptive statistics for BNSS items and subscales are presented in Table 2. The lack of normal distress as well as the quantity of speech items had skew $>1.0$ indicating distribution towards nonpathological ratings.

\section{Internal consistency}

Cronbach's alpha, computed to analyze internal consistency, was 0.93 , indicating that the items measure a distinct latent construct of negative symptoms. Item total correlations showed that all BNSS items were significantly correlated with the BNSS total scale score (Table 2). Alpha if-item-deleted coefficients ranged from 0.92 to 0.93 , with only the lack of normal distress item lowering the value.

\section{Inter-rater reliability}

To assess inter-rater reliability (Table 2), Intraclass Correlation Coefficients (ICCs) were calculated for the BNSS total score and for each subscale. The ICC for the BNSS total score was 0.97 , and ICC values for the subscales were: Anhedonia 0.88, distress 0.93 , asociality 0.95 , avolition 0.87 , blunted affect 0.95 , and alogia 0.97 . 
Table 2 Descriptive statistics, item-total correlations, ICC's

\begin{tabular}{|c|c|c|c|c|c|c|}
\hline & M & SD & Skew & Kurtosis & Item-total score r & $I^{\prime C C^{b}}$ \\
\hline \multicolumn{7}{|l|}{ Anhedonia } \\
\hline 1. Intensity of pleasure during activities & 2.3 & 1.6 & 0.1 & -0.9 & $0.80^{* *}$ & \\
\hline 2. Frequency of pleasurable activities & 2.5 & 1.6 & 0.0 & -0.8 & $0.78^{* *}$ & \\
\hline 3. Intensity of future pleasure & 2.1 & 1.5 & 0.4 & -0.3 & $0.69^{* *}$ & 0.88 \\
\hline Subscale total & 7.1 & 4.4 & 0.1 & -0.6 & & \\
\hline \multicolumn{7}{|l|}{ Distress subscale } \\
\hline 4. Lack of normal distress & 1.0 & 1.4 & $1.3^{\mathrm{a}}$ & 0.7 & $0.51^{* *}$ & 0.93 \\
\hline \multicolumn{7}{|l|}{ Asociality subscale } \\
\hline 5. Asociality behavior & 2.6 & 1.4 & 0.1 & -0.3 & $0.70^{* *}$ & \\
\hline 6. Asociality inner-experience & 2.0 & 1.4 & 0.6 & -0.3 & $0.71^{* *}$ & \\
\hline Subscale total & 4.6 & 2.6 & 0.4 & -0.3 & & 0.95 \\
\hline \multicolumn{7}{|l|}{ Avolition subscale } \\
\hline 7. Avolition behavior & 2.5 & 1.3 & 0.0 & -0.6 & $0.74^{* *}$ & \\
\hline 8. Avolition inner-experience & 2.1 & 1.5 & 0.2 & -0.7 & $0.79^{* *}$ & \\
\hline Subscale total & 4.7 & 2.7 & 0.2 & -0.5 & & 0.87 \\
\hline \multicolumn{7}{|l|}{ Blunted affect subscale } \\
\hline 9. Facial expression & 2.2 & 1.6 & 0.6 & -0.4 & $0.85^{* *}$ & \\
\hline 10. Vocal expression & 2.0 & 1.7 & 0.6 & -0.5 & $0.81^{* *}$ & \\
\hline 11. Expressive gestures & 2.1 & 1.7 & 0.4 & -1.0 & $0.78^{* *}$ & \\
\hline Subscale total & 6.3 & 4.7 & 0.6 & -0.5 & & 0.95 \\
\hline \multicolumn{7}{|l|}{ Alogia subscale } \\
\hline 12. Quantity of speech & 1.3 & 1.5 & $1.1^{\mathrm{a}}$ & 0.9 & $0.72^{* *}$ & \\
\hline 13. Spontaneous elaboration & 1.5 & 1.7 & 0.9 & 0.0 & $0.75^{* *}$ & \\
\hline Subscale total & 2.7 & 3.1 & 1.0 & 0.1 & & 0.97 \\
\hline BNSS total score & 26.3 & 14.7 & 0.7 & 0.1 & & 0.97 \\
\hline
\end{tabular}

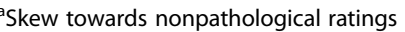

${ }^{\mathrm{b}} n=28$, Two-way mixed model, absolute agreement definition, average measures

${ }^{* *} p<0.01$

\section{Convergent validity with clinician-rated negative symptoms and functioning}

To assess convergent validity (Table 3 ) we used clinician rated scales (SANS, PANSS). The BNSS total was strongly correlated with the SANS Total $(r=0.89, p<0.001)$ and PANSS Negative factor (i.e., emotional withdrawal, poor rapport, passive/apathetic social withdrawal, lack of spontaneity and motor retardation) $(r=0.89, p<0.001)$, suggesting good convergent validity with other negative symptom measures.

Good subscale-level convergent validity was indicated by moderate to high correlations between BNSS subscale scores and the average of items comprising the 4 subscales of the SANS: BNSS anhedonia with SANS anhedonia/asociality $(r=0.58, p<0.001)$; BNSS asociality with SANS anhedonia/asociality $(r=0.79, p<0.001)$; BNSS avolition with SANS avolition $(r=0.79, p<0.001)$; BNSS blunted Affect with SANS blunted Affect $(r=0.93, p<0.001)$; and BNSS alogia with SANS alogia $(r=0.82, p<0.001)$.
The BNSS lack of normal distress item was negatively correlated with the PANSS depressed factor $(r=-0.33$, $p<0.001$ ), supporting the item's validity.

The BNSS total score had a high inverse correlation with the GAF total $(r=-0.69, p<0.001)$ score, as well as the PSP total score $(r=-0.70, p<0.001)$, suggesting good convergent validity with functional outcome. A partial correlation was conducted, controlling for depression, anxiety, psychosis and disorganization, to determine whether this association remained significant when accounting for common sources of secondary negative symptoms. The correlation between the BNSS total and the GAF total remained highly significant $(r=-0.62, p<0.001)$.

\section{Convergent validity with self-rated anhedonia and observer-rated apathy}

The TEPS self-report scale (Table 3) was used for convergent validity of the anhedonia subscale. The self-report scale was filled out by a smaller subsample 
Table 3 Convergent validity

\begin{tabular}{|c|c|c|c|c|}
\hline & BNSS total & BNSS motivation/pleasure & BNSS diminished expression & BNSS anhedonia \\
\hline PANSS negative factor & $0.89^{* *}$ & $0.71^{* *}$ & $0.90^{* *}$ & \\
\hline SANS avolition/apathy & $0.63^{* *}$ & $0.65^{* *}$ & $0.47^{* *}$ & $0.43^{* *}$ \\
\hline SANS asociality/anhedonia & $0.77^{* *}$ & $0.80^{* *}$ & $0.57^{* *}$ & $0.58^{* *}$ \\
\hline SANS blunted affect & $0,82^{* *}$ & $0.56^{* *}$ & $0.91^{* *}$ & \\
\hline SANS alogia & $0.63^{* *}$ & $0.37^{* *}$ & $0.77^{* *}$ & \\
\hline SANS total & $0.89^{* *}$ & $0.73^{* *}$ & $0.87^{* *}$ & \\
\hline AES total $(n=48)$ & $-0.48^{* *}$ & $-0.50^{* *}$ & $-0.40^{* *}$ & \\
\hline GAF & $-0.69^{* *}$ & $-0.68^{* *}$ & $-0.56^{* *}$ & \\
\hline \multirow[t]{2}{*}{ PSP total } & $-0.73^{* *}$ & $-0.73^{* *}$ & $-0.57^{* *}$ & \\
\hline & TEPS anticipatory & TEPS consummatory & & \\
\hline BNSS intensity of pleasure & $-0.25^{*}$ & $-0.29^{*}$ & & \\
\hline BNSS intensity of future pleasure & $-0.26^{*}$ & -0.20 & & \\
\hline BNSS anhedonia subscale & $-0.31^{*}$ & $-0.36^{*}$ & & \\
\hline SANS asociality/anhedonia & -0.13 & -0.15 & & \\
\hline
\end{tabular}

Note. ${ }^{*} p<0.05 ;{ }^{* *} p<0.01$

$(n=66)$. The BNSS anhedonia subscale was negatively correlated with both the anticipatory $(r=-0.31, p<0.05)$ and consummatory $(r=-0.36, p<0.001)$ pleasure scales from the TEPS. Regarding the attempt to discriminate between anticipatory and consummatory pleasure, the BNSS anticipatory $(r=-0.26, p<0.05)$ as well as the BNSS intensity of pleasure item $(r=-0.25, p<0.05)$ were correlated with the TEPS anticipatory pleasure scale. On the other hand, only the BNSS consummatory $(r=-0.29$, $p<0.05)$ but not the anticipatory item $(r=-0.20, p>0.05)$ was correlated with the TEPS consummatory pleasure scale. However, the two correlation coefficients were not significantly different. The SANS anhedonia-asociality scale was neither correlated with TEPS anticipatory pleasure scale $(r=-0.13, p>0.05)$ nor the consummatory pleasure scale $(r=-0.15, p>0.05)$. These results support the convergent validity of the BNSS anhedonia items.

The AES observer scale (Table 3) was completed by the inpatient's primary nurse $(n=49)$ and correlated moderately with the BNSS total score $(r=0.48, p<0.001)$ and the BNSS motivation/pleasure scale (i.e., average of anhedonia, avolition, asociality items) $(r=0.50, p<0.001)$. Thus, BNSS ratings correspond well to apathy ratings by the primary care person.

\section{Discriminant validity}

Discriminant validity (Table 4) of the BNSS was examined by calculating correlations with positive symptoms, disorganized symptoms, general symptoms, and extrapyramidal side effects (MSAS). BNSS total was not significantly correlated with the PANSS positive factor $(r=-0.02, p>0.05)$, PANSS depressed factor $(r=0.02, p>0.05)$ or PANSS excited factor $(r=0.19, p>0.05)$. There was a modest correlation between the PANSS disorganized factor and the BNSS total score $(r=0.37, p<0.001)$. This correlation was mainly due to the Expression subscales (range $r=0.40-0.42, p<0.01)$, whereas no correlation with the subscales of the Motivation and Pleasure factor was observed (range $r=-0.04-0.23, p>0.05$ ). However, the mean score on the disorganization factor was 5.00 (2.12) (lowest possible 3 ) and the maximum score 10 (from 21 possible), therefore representing a nondisorganized sample. The CDSS total score was not significantly correlated with the BNSS total score $(r=0.16$, $p>0.05)$ or the BNSS anhedonia score $(r=0.08, p>0.05)$. Finally, the BNSS total score was not correlated with the MSAS $(r=0.12, p>0.05)$.

\section{Discussion}

Overall the German translation of the BNSS shows good psychometric properties including inter-rater reliability, which mostly replicate the findings for the original version. Importantly, we show that overall the BNSS anhedonia subscales converge with self-reported anhedonia, although the differentiation between anticipatory and consummatory anhedonia remains a challenge. In addition, BNSS rated negative symptoms correlate with observerrated apathy as assessed by the primary nurse.

Convergent validity with the SANS total and the PANSS negative factor was high and moderate to high for the respective subscales. It has to be noted that the observed correlations might be somewhat higher than expected, because the same rater scored the BNSS and the other negative symptom scales. Nevertheless, these results confirm the assumption of a common and established construct for negative symptoms. We also found strong associations between negative symptoms and functioning, emphasizing the importance of its role as a predictor of poor outcome [2]. 
Table 4 Discriminant validity

\begin{tabular}{lccccc}
\hline & PANSS positive & PANSS depressed & PANSS disorganized & PANSS excited & CDSS $^{\text {MSAS }^{a}}$ \\
\hline BNSS total & -0.02 & 0.02 & $0.37^{* *}$ & 0.19 & 0.16 \\
SANS total & 0.07 & 0.14 & $0.42^{* *}$ & $0.29^{*}$ & 0.12 \\
PANSS negative & -0.01 & 0.16 & $0.41^{* *}$ & $0.30^{* *}$ & 0.10 \\
BNSS anhedonia & -0.13 & -0.05 & 0.23 & 0.07 & 0.26 \\
\hline
\end{tabular}

${ }^{a} n=56$

${ }^{*} p<0.05$; ${ }^{* *} p<0.01$

For anhedonia, we could show incremental validity as the TEPS scales only correlated with the BNSS anhedonia scale, but not with the SANS anhedonia subscale. This strongly suggests that BNSS anhedonia captures the subjective experience of anhedonia better than older negative symptom scales. The importance of the distinction between anticipatory and consummatory pleasure has been emphasized, as only the former has been consistently found to be impaired in patients with schizophrenia [25]. We also addressed the question of whether the individual BNSS anhedonia items show specific correlations with the respective TEPS anticipatory and consummatory subscales. However, this distinction was limited, as all correlation coefficients were in a similar range. Only the BNSS intensity of pleasure item seemed to be somewhat more strongly related to the TEPS consummatory anhedonia subscale, but differences between correlations were small and non-significant. The lack of convergent validity between the TEPS subscales and BNSS anhedonia items could reflect limitations of the BNSS, TEPS, or both measures in evaluating consummatory and anticipatory pleasure. The TEPS consummatory and anticipatory pleasure subscales have shown somewhat diverging findings across studies, with some investigations showing a deficit in consummatory but not anticipatory [29] and others an impairment in anticipatory but not consummatory pleasure [25]. This raises the question whether the TEPS was fully suited for assessing the specific convergent validity of the items with the anhedonia subscale. Also, the BNSS intensity item may show restricted range and rely on memory aspects that might be impaired in patients, suggesting that few patients may have a true consummatory pleasure deficit [30]. Thus, it remains an open question whether the current convergent validity findings on anhedonia reflect limitations of the scales or the conceptualization of anhedonia as a consummatory vs. anticipatory pleasure deficit in schizophrenia.

To expand prior work on convergent validity to a different assessment modality, we included the AES as an observer rating of apathy that was completed by the patient's primary nurse. We found moderate correlations between AES ratings and BNSS total and motivation/ pleasure scores. This is quite remarkable, because the nurses were not trained in the assessment of negative symptoms and were simply instructed to score the AES based on their observations in daily life on the ward. Interview-based assessments always raise the question whether the participant correctly reports his interests and activities [31], but the present findings suggest a good convergence between interview-based reports and observer ratings. Whether this convergence generalizes to non-professional care-givers such as relatives remains an open issue.

Discriminant validity was shown by excellent differentiation between the BNSS and measures of positive symptoms and depression. It can therefore be concluded that the BNSS clearly measures disturbances that are different from other symptom dimensions of schizophrenia. In addition, extrapyramidal side effects have been considered as major cause for secondary negative symptoms. The only study with the BNSS to have previously included a measurement of extrapyramidal symptoms has taken a categorical approach of present or absent extrapyramidal side effects [16]. Here, we have employed a dimensional approach, which shows that BNSS total and subscales were not associated with extrapyramidal symptoms. This is in line with the categorical findings by Mucci and colleagues, thereby supporting discriminant validity.

Some limitations of the present study need to be considered. First, overall the participants showed relatively low levels of positive, depressive, and extrapyramidal symptoms. Therefore, it is an important question whether the excellent discriminant validity reported here generalizes to populations with higher non-negative symptom levels. Third, the inclusion criteria may impact the ability of these findings to generalize to samples with greater stability and comorbid substance use disorders. Finally, the interpretation of convergent validity analyses is complicated by the use of different different forms of assessment (i.e., self-report questionnaires vs. clinician rated) and different types of raters (e.g., nurse vs. clinician). As such, method and rater variance should be accounted for when interpreting the correlations between BNSS scores and measures of anhedonia and apathy.

\section{Conclusions}

The outcome of this study suggest that the BNSS can be used to assess negative symptoms across different languages 
and health care systems. Furthermore, our data show that convergent validity extends to self-ratings of anhedonia and observer-ratings of apathy. Overall, the BNSS is a promising instrument for the investigation of pathophysiological mechanisms underlying the negative symptoms of schizophrenia and particularly the two dimensions apathy/ avolition and diminished expression. Most importantly, it allows assessment of negative symptoms across cultures and thus could possibly be of use in future international multicenter trials that target negative symptoms.

\begin{abstract}
Abbreviations
AES: Apathy evaluation scale; BNSS: Brief negative symptom scale;

CDSS: Calgary depression scale for Schizophrenia; CAINS: Clinical assessment interview for negative symptoms; DSM-IV: Diagnostic and statistical manual of mental disorders, fourth edition; GAF: Global assessment of functioning scale; ICC: Intraclass correlation coefficient; MSAS: Modified simpson-angus scale; PANSS: Positive and negative syndrome scale; PSPS: Personal and social performance scale; SANS: Scale for the assessment of negative symptoms; TEPS: Temporal experience pleasure scale
\end{abstract}

\section{Acknowledgements}

The authors would like to thank Benedikt Habermayer, MD, for his help to recruit patients.

\section{Funding}

The study was funded in part by grants from the Swiss National Science Foundation (Grant No 105314_130351) and the Volkswagen Foundation (Grant No 85 061) to the senior author. The funding sources had no influence on study design; in the collection, analysis and interpretation of data; in the writing of this manuscript; and in the decision to submit the article for publication.

\section{Availability of data and materials}

Available upon request to the first author Martin Bischof: martin.bischof@puk.zh.ch

\section{Authors' contributions}

$M B$, SK and GS designed the study. SK (PI) obtained funding for this study. $\mathrm{CO}$ conducted the German translation of the scale. $\mathrm{MB}, \mathrm{MH}, \mathrm{OH}, \mathrm{MK}$ and $\mathrm{AK}$ were involved in recruitment and assessment. MB performed statistical analyses. MB, SK and GS conducted the interpretation of the results and prepared the first draft of the manuscript. All authors contributed to and approved the final manuscript.

\section{Competing interests}

The authors declare that they have no competing interests.

\section{Consent for publication}

All participants were informed that the results of this study would be published in one or more publications and that their identities would not be made public in these publications. All participants agreed and gave their written consent.

\section{Ethics approval and consent to participate}

The study was approved by the Cantonal Ethics Committee of Zurich, and all participants gave written informed consent. Each participant was asked for their consent to participate before collecting any data.

\section{Author details}

'Department of Psychiatry, Psychotherapy and Psychosomatics, Psychiatric Hospital, University of Zurich, Lenggstrasse 31, 8032 Zurich, Switzerland. ${ }^{2}$ Laboratory for Social and Neural Systems Research, Department of Economics, University of Zurich, Bluemlisalpstrasse 10, 8006 Zurich, Switzerland. ${ }^{3}$ Department of Psychology, State University of New York at Binghamton, Binghamton, NY 13902-6000, USA.
Received: 19 May 2016 Accepted: 7 November 2016

Published online: 22 November 2016

\section{References}

1. Blanchard JJ, Cohen AS. The structure of negative symptoms within schizophrenia: implications for assessment. Schizophr Bull. 2006;32:238-45.

2. Rabinowitz J, Levine SZ, Garibaldi G, Bugarski-Kirola D, Berardo CG, Kapur S. Negative symptoms have greater impact on functioning than positive symptoms in schizophrenia: analysis of CATIE data. Schizophr Res. 2012;137:147-50.

3. Hunter R, Barry S. Negative symptoms and psychosocial functioning in schizophrenia: neglected but important targets for treatment. Eur Psychiatry. 2012;27:432-6

4. Fervaha G, Foussias G, Agid O, Remington G. Amotivation and functional outcomes in early schizophrenia. Psychiatry Res. 2013;210:665-8.

5. Marder SR, Daniel DG, Alphs L, Awad AG, Keefe RSE. Methodological issues in negative symptom trials. Schizophr Bull. 2011;37:250-4.

6. Foussias $\mathrm{G}$, Siddiqui I, Fervaha G, Agid O, Remington G. Dissecting negative symptoms in schizophrenia: Opportunities for translation into new treatments. J Psychopharmacol. 2015;29:116-26.

7. Lincoln, TM, Dollfus, S, Lyne, J. Current developments and challenges in the assessment of negative symptoms. Schizophrenia Research 2016; Epub ahead of print.

8. Andreasen NC. Negative symptoms in schizophrenia. Definition and reliability. Arch Gen Psychiatry. 1982;39:784-8.

9. Kirkpatrick B, Fenton WS, Carpenter WT, Marder SR. The NIMH-MATRICS consensus statement on negative symptoms. Schizophr Bull, vol. 32, Oxford University Press; 2006, pp. 214-9.

10. Messinger JW, Trémeau F, Antonius D, Mendelsohn E, Prudent V, Stanford $A D$, et al. Avolition and expressive deficits capture negative symptom phenomenology: implications for DSM-5 and schizophrenia research. Clin Psychol Rev. 2011;31:161-8.

11. Forbes C, Blanchard JJ, Bennett M, Horan WP, Kring A, Gur R. Initial development and preliminary validation of a new negative symptom measure: the Clinical Assessment Interview for Negative Symptoms (CAINS). Schizophr Res. 2010;124:36-42.

12. Kirkpatrick B, Strauss GP, Nguyen L, Fischer BA, Daniel DG, Cienfuegos A, et al. The brief negative symptom scale: psychometric properties. Schizophr Bull. 2011;37:300-5.

13. Strauss GP, Keller WR, Buchanan RW, Gold JM, Fischer BA, McMahon RP, et al. Next-generation negative symptom assessment for clinical trials: validation of the Brief Negative Symptom Scale. Schizophr Res. 2012;142:88-92.

14. Strauss GP, Hong LE, Gold JM, Buchanan RW, McMahon RP, Keller WR, et al. Factor structure of the brief negative symptom scale. Schizophr Res. 2012:142:96-8.

15. Mane A, Garcia-Rizo C, Garcia-Portilla MP, Berge D, Sugranyes G, GarciaAlvarez L, Fernandez-Egea E. Spanish adaptation and validation of the Brief Negative Symptoms Scale. Compr Psychiatry. 2014;55(7):1726-9.

16. Mucci A, Galderisi S, Merlotti E, Rossi A, Rocca P, Bucci P, et al. The Brief Negative Symptom Scale (BNSS): Independent validation in a large sample of Italian patients with schizophrenia. Eur Psychiatry 2015. In press.

17. Kay SR, Fiszbein A, Opler LA. The positive and negative syndrome scale (PANSS) for schizophrenia. Schizophr Bull. 1987;13:261-76.

18. Sheehan DV, Lecrubier $Y$, Sheehan KH, Amorim P, Janavs J, Weiller E, et al. The Mini-International Neuropsychiatric Interview (M.I.N.I.): the development and validation of a structured diagnostic psychiatric interview for DSM-IV and ICD-10. J Clin Psychiatry. 1998;59 Suppl 20:22-33. quiz34-57.

19. BFS. Medizinische Statisik der Krankenhäuser: Anzahl Fälle und durchschnittliche Aufenthalsdauer (DAD) nach Altersklasse und Diagnosekode. Neuchatel, Switzerland: BFS; 2012.

20. Frances A, Pincus HA, First MB. Global Assessment of Functioning Scale (GAF). Diagnositv and Statistical Manual of Mental Disorders. $4^{\text {th }}$ ed. Washington, DC: American Psychiatric Association; 1994.

21. Morosini PL, Magliano L, Brambilla L, Ugolini S, Pioli R. Development, reliability and acceptability of a new version of the DSM-IV Social and Occupational Functioning Assessment Scale (SOFAS) to assess routine social functioning. Acta Psychiatr Scand. 2000;101:323-9.

22. Addington D, Addington J, Schissel B. A depression rating scale for schizophrenics. Schizophr Res. 1990;3:247-51.

23. Wallwork RS, Fortgang R, Hashimoto R, Weinberger DR, Dickinson D. Searching for a consensus five-factor model of the positive and negative syndrome scale for schizophrenia. Schizophr Res. 2012;137:246-50. 
24. Marin RS, Biedrzycki RC, Firinciogullari S. Reliability and validity of the Apathy Evaluation Scale. Psychiatry Res. 1991;38:143-62.

25. Gard DE, Kring AM, Gard MG, Horan WP, Green MF. Anhedonia in schizophrenia: Distinctions between anticipatory and consummatory pleasure. Schizophr Res. 2007;93:253-60.

26. Simpson GM, Angus JW. A rating scale for extrapyramidal side effects. Acta Psychiatr Scand Suppl. 1970;212:11-9.

27. Lee IA, Preacher KJ. Calculation for the test of the difference between two dependent correlations with one variable in common [Computer software]. 2013. Available from http://quantpsy.org/corrtest/corrtest2.htm. Accessed Sept 2013.

28. Steiger JH. Tests for comparing elements of a correlation matrix. Psychol Bull. 1980;87:245-51.

29. Strauss GP, Wilbur RC, Warren KR, August SM, Gold JM. Anticipatory vs. consummatory pleasure: What is the nature of hedonic deficits in schizophrenia? Psychiatry Res. 2011;187:36-41.

30. Strauss GP, Gold JM. A new perspective on anhedonia in schizophrenia. Am J Psychiatry. 2012;169:364-73.

31. Horan WP, Kring AM, Blanchard JJ. Anhedonia in Schizophrenia: a review of assessment strategies. Schizophr Bull. 2006;32:259-73.

Submit your next manuscript to BioMed Central and we will help you at every step:

- We accept pre-submission inquiries

- Our selector tool helps you to find the most relevant journal

- We provide round the clock customer support

- Convenient online submission

- Thorough peer review

- Inclusion in PubMed and all major indexing services

- Maximum visibility for your research

Submit your manuscript at www.biomedcentral.com/submit
Biomed Central 